\title{
Increased susceptibility of Huh7 cells to HCV replication does not require mutations in RIG-I
}

\author{
Dino A Feigelstock ${ }^{1 *}$, Kathleen B Mihalik', Gerardo Kaplan², Stephen M Feinstone ${ }^{1}$
}

\begin{abstract}
Background: The cytosolic retinoic acid-inducible gene I (RIG-I) is a pattern recognition receptor that senses HCV double-stranded RNA and triggers type I interferon pathways. The clone Huh7.5 of human hepatoma Huh7 cells contains a mutation in RIG-I that is believed to be responsible for the improved replication of HCV in these cells relative to the parental strain. We hypothesized that, in addition to RIG-I, other determinant(s) outside the RIG-I coding sequence are involved in limiting HCV replication in cell culture. To test our hypothesis, we analyzed Huh7 cell clones that support the efficient replication of HCV and analyzed the RIG-I gene.

Results: One clone, termed Huh7D, was more permissive for HCV replication and more efficient for HCV-neomycin and HCV-hygromycin based replicon colony formation than parental Huh7 cells. Nucleotide sequence analysis of the RIG-I mRNA coding region from Huh7D cells showed no mutations relative to Huh7 parental cells.

Conclusions: We derived a new Huh7 cell line, Huh7D, which is more permissive for HCV replication than parental Huh7 cells. The higher permissiveness of Huh7D cells is not due to mutations in the RIG-I protein, indicating that cellular determinants other than the RIG-I amino-acid sequence are responsible for controlling HCV replication. In addition, we have selected Huh7 cells resistant to hygromycin via newly generated HCV-replicons carrying the hygromycin resistant gene. Further studies on Huh7D cells will allow the identification of cellular factors that increased the susceptibility to HCV infection, which could be targeted for anti-HCV therapies.
\end{abstract}

\section{Background}

Hepatitis C virus (HCV) infects nearly 200 million people worldwide [1]. HCV infection causes chronic liver disease, cirrhosis, and is associated with hepatocellular carcinoma [2]. It is estimated that only $15-40 \%$ of infected people resolve acute HCV infection [3], suggesting that host factors are capable of controlling $\mathrm{HCV}$ replication in some individuals. However, the host determinants responsible for controlling $\mathrm{HCV}$ replication are not well understood. The ability to grow $\mathrm{HCV}$ in vitro is important for understanding both virologic and immunologic aspects of $\mathrm{HCV}$ infections. It has been shown that the JFH1 and the chimeric J6/JFH1 isolate of the 2a genotype of HCV replicate efficiently in Huh7 cells $[4,5]$ and in the highly permissive Huh7.5 and Huh7.5.1 cells derived from the human hepatoma cell line Huh7 [6-9]. Later, production of infectious genotype $1 \mathrm{a}$ and $1 \mathrm{~b}$ viruses [10] was demonstrated in Huh7.5 cells. Further studies showed that the increased permissiveness of Huh7.5 cells results from a mutation (Thr-55-Iso) in the RIG-I gene (retinoic acid-inducible gene I, a DExD/H domain containing RNA helicase, reviewed in [11]) which impairs interferon signaling [12]. In this study, using a technique similar to that used to generate the Huh7.5 cell line, we derived another Huh7 cell line highly permissive for HCV replication that we termed Huh7D. We compared the replication of the genotype 2a J6/JFH1 strain of HCV and the genotype $1 \mathrm{~b}$ based $\mathrm{HCV}$ replicons in Huh7D cells with replication in Huh7 and Huh7.5 cells. We found that while HCV replicated better in Huh7D cells relative to Huh7 cells, no mutations were found in the RIG-I coding region from Huh7D cells, indicating that cellular determinants located outside the RIG-I amino-acid coding sequence are responsible for the higher permissiveness of Huh7D cells for HCV replication.

* Correspondence: dino.feigelstock@fda.hhs.gov

1 Division of Viral Products, Center for Biologics Evaluation and Research,

FDA, 29 Lincoln Drive, Bethesda, MD 20892, USA

(C) 2010 Feigelstock et al; licensee BioMed Central Ltd. This is an Open Access article distributed under the terms of the Creative Commons Attribution License (http://creativecommons.org/licenses/by/2.0), which permits unrestricted use, distribution, and reproduction in any medium, provided the original work is properly cited. 


\section{Results \\ Huh7D cells are more susceptible to HCV replicons than parental Huh7 cells}

To compare the susceptibility of cells to HCV replicons, Huh7, Huh7D, and Huh7.5 cells were transfected with HCV-neo-Replicon and selected with $250 \mu \mathrm{g} / \mathrm{ml}$ of G418. An increased number of neomycin-resistant colonies were observed in Huh7D cells (and control Huh7.5 cells) relative to Huh7 cells (figure 1), irrespective of the amount of transfected RNA. No surviving colonies were observed in replication-defective HCV-neo-Replicon, unrelated RNA (transcribed from pTRI-Xef plasmid from AMBION kit), or no RNA transfected cells (figure 1 and additional file 1 ). In order to quantify the efficiency of colony formation (ECF), we repeated the experiment using lower amounts of replicon, and obtained ECF of 526, 10,500, and 2631 colonies per $\mu \mathrm{g}$ of transfected RNA for Huh7, Huh7D, and Huh7.5 cells respectively (additional file 1 ). Wild type HCV-neo replicons obtained by in vitro transcription of Sca1 cut plasmid pFK i389neoNS3-3'/WT and other less adapted replicons (Feigelstock et al, unpublished) also yielded more colonies in Huh7D cells relative to Huh7 cells. These results show Huh7D cells have an increased capacity to survive G-418 via HCV-neo-Replicon than parental Huh7 cells, suggesting that the HCV replicon replicates better in Huh7D cells relative to Huh7 cells.

The increased susceptibility of Huh7D cells to HCVreplicons is independent of the selectable marker coded by the HCV-replicon

To determine whether the selectable marker contained in the HCV-replicon had an effect in the susceptibility of the Huh7 cell clones, we transfected Huh7, Huh7D, and Huh7.5 cells with approximately $100 \mathrm{ng}$ of the indicated HCV-hyg-Replicons and selected cells with $65 \mu \mathrm{g} /$ $\mathrm{ml}$ hygromycin B. At 40 days post-transfection, more hygromycin B resistant colonies were observed in Huh7D and Huh7.5 cells than in Huh7 cells whereas mock-transfected cells did not survive the antibiotic selection (figure $2 \mathrm{a}$ ). As shown in figure $2 \mathrm{a}$, we were able to select Huh7 colonies resistant to hygromycin B; however, those initially resistant colonies didn't survive longer (more than 60 days) treatment with hygromycin B. Replication of HCV-hyg-Replicon in Huh7D and Huh7.5 cells was confirmed by immunfluorscence analysis (figure 2b). Transfection with HCV-hyg-Replicons yielded a low number of surviving colonies and, given the extended time required for hygromycin to kill Huh7 cells, we needed to make a cell passage resulting in the loss of our ability to accurately quantify the differences in transduction efficiencies. These data indicate that the selectable marker has no effect in the higher susceptibility of the Huh7 clones to HCV replicons.

\section{Huh7D cells are more susceptible to HCV infection than Huh7 parental cells}

We next wanted to determine whether Huh7D cells were more susceptible to HCV replication than parental Huh7 cells when using the J6/JFH1 infectious clone. To do so, we infected Huh7, Huh7D, and Huh7.5 cells with HCV-J6/JFH1 at an m.o.i. of 0.01 and analyzed virus growth at $0,1,3,5,7,10$, and 15 dpi using an IF endpoint dilution titration assay and by IF on infected cells. HCV J6/JFH1 grew faster in Huh7D and Hu7.5 cells relative to Huh7 cells (figure $3 \mathrm{a}$ ), which is consistent with our results showing that the Huh7D and Huh7.5 cells were more susceptible to HCV replicons than the parental Huh7 cells. Cells passed to 96 well plates were stained with anti-HCV antibodies at 3, 5, and $10 \mathrm{dpi}$, and $\mathrm{HCV}$ antigen was detected by IF analysis (figure $3 \mathrm{~b})$. In agreement with the titration data, Huh7D and Huh7.5 cells showed an increase in the percentage of infected cells relative to Huh7 cells. These results show that J6/JFH1 virus grew better in Huh7D cells and in Huh7.5 cells than in Huh7 cells. In order to discard the possibility that the J6/JFH1 virus grew better in Huh7D cells relative to Huh7 and Huh7.5 cells because it was produced in Huh7D cells (and therefore may have acquired Huh7D adaptive mutations), we sequenced the full length genome of the J6/JFH1 virus we used to inoculate the cells. We found no differences in the nucleotide sequence with respect to the J6/JFH1 sequence present in the plasmid, except in three positions that showed a mixture of two nucleotides (T2667T/C; A7150G/A; and T7667T/A). In addition, we repeated the experiment shown in figure 3 but using a J6/JFH1 virus that had been grown only in Huh7.5 cells and therefore there was no chance that the virus had adapted to the Huh7D cells prior to studying the replication of the virus in those cells. Again we saw higher titers in Huh7D relative to Huh7 cells (2 logs). This result suggest that the observed higher susceptibility of Huh7D cells to J6/JFH1 infection is not due to adaptation of the virus to Huh7D cells. Furthermore, JFH1 virus (also not passaged in Huh 7D cells) also grew better in Huh7D and Huh7.5 cells relative to Huh7 cells (not shown).

\section{There are no amino-acid substitutions in the RIG-I coding region from Huh7D cells}

In order to determine if the increased susceptibility of Huh7D cells to HCV replication was due to mutations in RIG-I as observed in Huh7.5 cells [12], we sequenced 


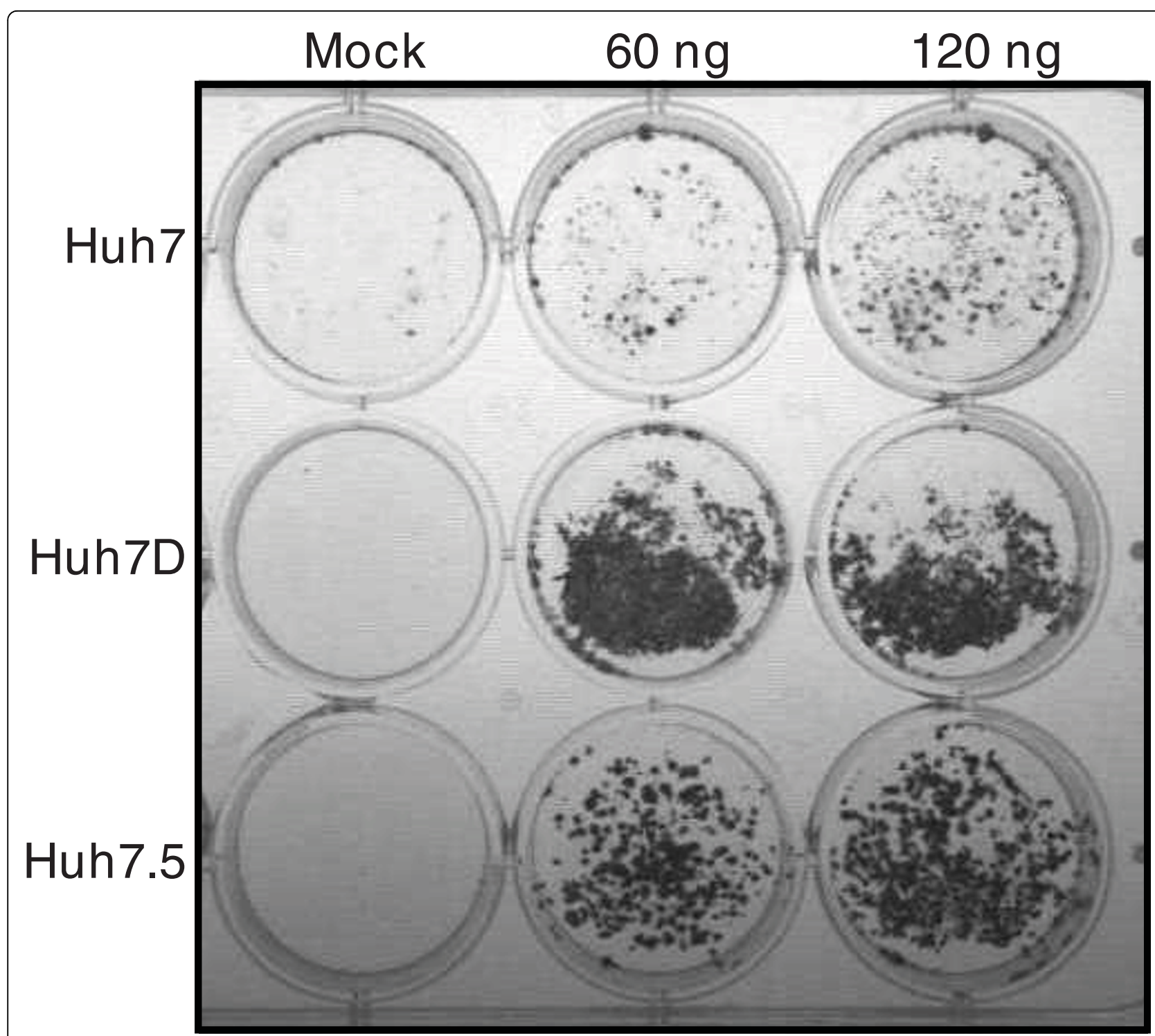

Figure 1 Transfection of HCV-neo-replicon into Huh7, Huh7D, and Huh7.5 cells. Coomassie staining of Huh7, Huh7D, and Huh7.5 cells that were transfected with the indicated amounts of HCV-neo replicon and selected for 13 days with G-418 at a concentration of $250 \mu \mathrm{g} / \mathrm{ml}$.

the full coding region of the RIG-I mRNA from Huh7, Huh7D, and Huh7.5 cells by RT-PCR. The RIG-I coding region sequence was identical in Huh7D and parental Huh7 cells while the expected ACA to ATA (Thr-55Iso) substitution was found in Huh7.5 cells (additional file 2).

\section{Discussion}

In this study we derived Huh7D cells, a single cell clone of replicon-cured Huh7 cells. We show that as the previously reported Huh7.5 cells, Huh7D cells are more permissive to $\mathrm{HCV}$ replication than parental Huh7 cells. Huh7D cells were similarly permissive to HCV replicon (neo and hyg) replication as Huh7.5 cells, and were at least as permissive to $\mathrm{HCV} \mathrm{J6/JFH1} \mathrm{infection} \mathrm{as} \mathrm{Huh7.5}$ cells. Sequencing of the coding region of RIG-I mRNA from Huh7D cells, as opposed to the RIG-I coding region from Huh7.5 cells, showed no mutations when compared to the RIG-I coding region from parental Huh7 cells. This indicates that mutations in RIG-I coding region are not responsible for the higher permissiveness of Huh7D cells to HCV replication. This is in agreement with recent observations indicating that an intact RIG-I signaling pathway does not necessarily limit HCV replication in Huh-7 cells [13].

At this time we have not identified the factor/s responsible for the higher permissiveness of Huh7D cells. Other than RIG-I cellular factors affecting HCV 


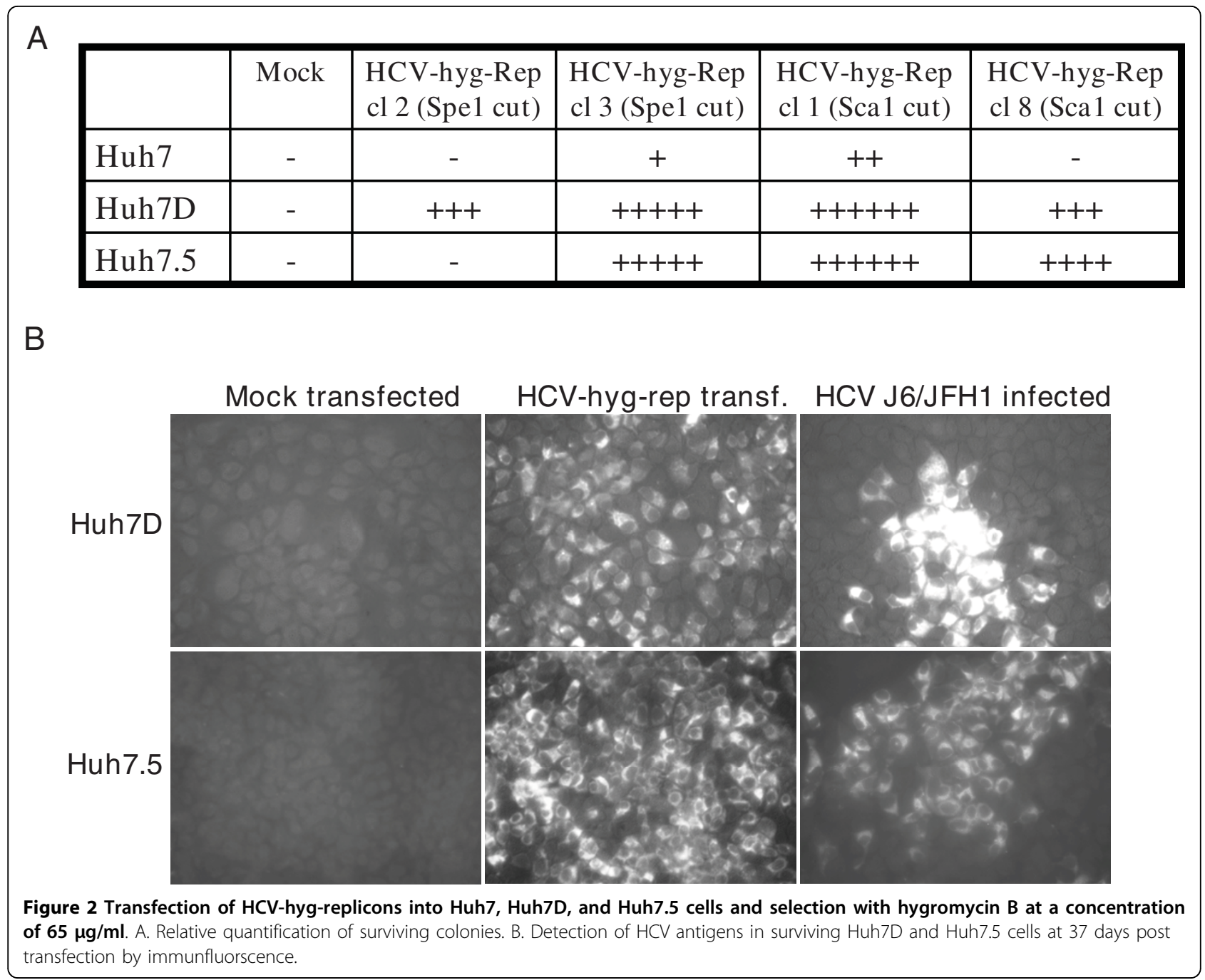

replication have been identified. Reconstituted Toll like receptor 3 (TLR3) in Huh7 and Huh7.5 cells senses $\mathrm{HCV}$ infection independently of RIG-I, and triggers an antiviral state [14]. Class III Phosphatidylinositol 4Kinase alpha and beta were recently identified as regulators of hepatitis $C$ virus replication in Huh7 cells [15]. A screening using siRNA identified host genes that modulate $\mathrm{HCV}$ replication, including host genes related to the RNAi pathway [16], transcription factors, transporter proteins, and others [17].

In order to obtain a Huh7 cell line with even higher permissiveness for HCV replication, we selected double cured Huh7 cells (cells selected with HCV-neo replicon, cured, selected with HCV-hyg replicon, and cured again, or cells selected twice with HCV-neo replicons), but we couldn't obtain Huh7 cells with higher permissiveness for HCV replicon replication or HCV infection (not shown). The failure to obtain cells that are more permissive to $\mathrm{HCV}$ replication by successive curing of transfected cells suggests that cellular mechanisms involved in HCV replication are difficult to alter. It is also possible that interferon signaling is the major cellular mechanism for controlling HCV replication (and/or the easiest to alter), and once this pathway is altered, few other (alterable) pathways are left to facilitate HCV replication.

We have shown that Huh7D cells are more permissive than Huh7 cells not only for a replicon with the neo selectable marker, but also for an $\mathrm{HCV}$ replicon which expresses the hygromycin resistance gene. We were able to select Huh7D (and Huh7.5) but not Huh7 cells resistant to hygromycin B after HCV-hyg replicon transfection. Although we were able to initially select Huh7 cells resistant to hygromycin $\mathrm{B}$, treatment with the antibiotic for periods longer than 60 days induced the extinction of the Huh7 colonies. This observation suggest that replication of HCV-hyg-rep in Huh7 cells is limited, but the lesser susceptibility of Huh7 cells to 


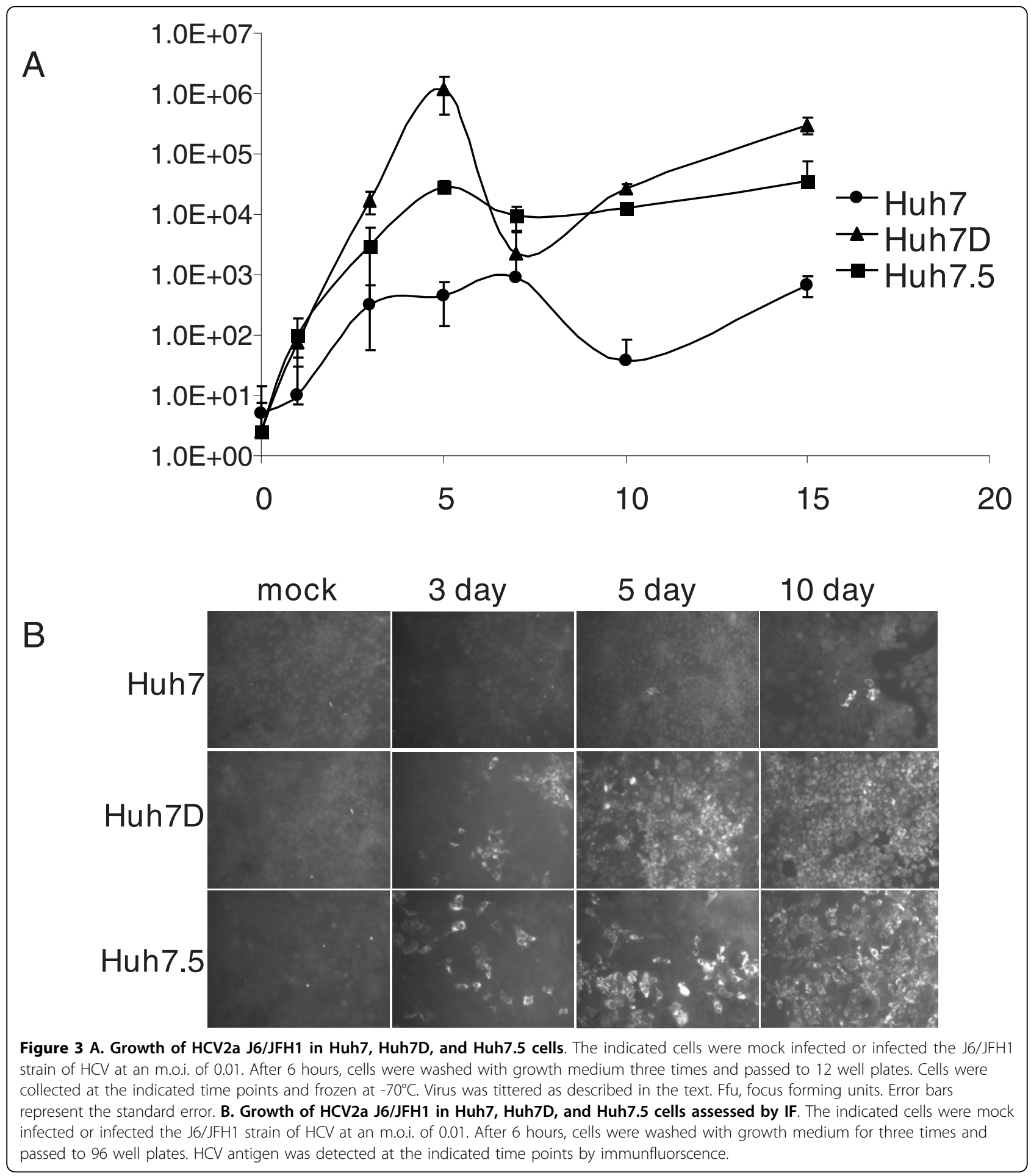

hygromycin permits longer surviving of the colonies. In fact, while mock transfected Huh7 cells survive $250 \mu \mathrm{g} /$ $\mathrm{ml}$ Neomycin for about 2 weeks, mock transfected Huh7 cells survive $65 \mu \mathrm{g} / \mathrm{ml}$ hygromycin for about 30 days. Obtaining resistance to hygromycin B was more difficult and less efficient than obtaining resistance to neomycin. We don't know the reason underlying this observation, which together with the fact that resistance of Huh7 cells to hygromycin B via HCV-hyg replicons was not widely reported, suggest the presence of intrinsic barriers for Huh7 cells to survive hygromycin B using HCV-hyg replicons. 


\section{Conclusion}

In this study we derived a new Huh7 cell line (Huh7D) which is more permissive for $\mathrm{HCV}$ replication than parental Huh7 cells. The permissiveness of Huh7D cells is not due to mutations in the RIG-I protein, as reported for the widely used Huh7.5 cells. More experiments are needed to elucidate if the cellular determinant/s responsible for the higher permissiveness of Huh7D cells are related to the interferon or other cellular pathways.

\section{Methods}

\section{Cells}

Huh7 and Huh7.5 cells were a gift from Jake Liang. Huh7, Huh7D, and Huh7.5 cells were grown in DMEM (Gibco) supplemented with $10 \%$ bovine calf serum (Atlanta Biologicals), L-glutamine (Gibco), penicillin and streptomycin (Gibco).

\section{Viruses}

The JFH1 virus was a gift from Takaji Wakita. J6/JFH1 virus was obtained by transfection of Huh7D cells (see below) with in vitro transcribed HCV J6/JFH1 RNA. HCV J6/JFH1 RNA was obtained from plasmid pFL- J6/ JFH1 (a gift from Charles Rice) that was cut with Xba1 and transcribed with T7 RNA polymerase (T7 Megascript AMBION).

\section{Generation of $\mathrm{HCV}$ replicon containing the neomycin resistance gene ("HCV-neo-Rep")}

HCV-neo-Replicon and replication-defective HCV replicon were obtained as previously described [18]. Briefly, plasmid pFK i389neoNS3-3'/NK5.1 coding for a highly permissive HCV-neo replicon harboring several replication enhancing mutations [19] and plasmid pFK i389neoNS3-3'/delta5B [18] (kindly provided by Ralph Bartenschlager) were cut with restriction enzyme Sca1, and in vitro transcribed using T7 Megascript kit (AMBION).

\section{Generation of HCV replicon containing the hygromycin resistance gene ("HCV-hyg-Rep")}

To obtain HCV replicons carrying the hygromycin resistance gene ("HCV-hyg-Rep") we replaced the neomycin resistance gene with the hygromycin resistance gene in plasmid pFK i389neoNS3-3'/NK5.1 using restriction enzymes Asc1 and Pme1. Hygromycin resistance gene was obtained by PCR using plasmid pIREShyg (Clontech) as template and sense oligo AACTAAAGGCGCGCCATGGATAGATCCGGAAAGCCTGAACTCAC (carrying the Asc1 restriction site) and anti-sense oligo AGTTATGGTTTAAACCTATTCC TTTGCCCTCGGACGAGTGCTGGG or anti-sense oligo AGTTATGGTTTAAACCTATTCCTTTGC
CCTCGGACGAGTGCTGGGGCGTCGGTTTCCACTATCGGCGAGAACTTCTAC (both carrying the Pme1 restriction site). The later anti-sense oligo is designed to mutate the Sca1 restriction site present at the 3' end of the hygromycin resistant gene, without changing the coded amino-acid (restriction enzyme Sca1 is used to linearize the vector in order to make in vitro transcripts, see below). The PCR products were cut with restriction enzymes Asc1 and Pme1 and ligated to plasmid pFK i389neoNS3-3'/NK5.1 that was cut with same restriction enzymes to obtain pFK i389hygNS3-3'/NK5.1 and pFK i389hygscalessNS3-3'/NK5.1. The resultant recombinant plasmids were transformed into TOP10 competent bacteria (Invitrogen). Bacteria clones carrying the HCVhyg-Replicons were confirmed by restriction analysis and sequencing. Plasmids pFK i389hygNS3-3'/NK5.1 (clones 2 and 3 ) were cut with restriction enzyme Spe 1 (generating a replicon with additional 4 nucleotides at the 3' end) and plasmids pFK i389hygscalessNS3-3'/ NK5.1 (clones 1 and 8) were cut with Sca1 (to obtain a replicon with the authentic 3' end sequence), and in vitro transcribed using T7 Megascript kit (AMBION).

\section{Generation of Huh7D cells}

Huh7 cells grown in 12 well plates were transfected with approximately 100 or $200 \mathrm{ng}$ of HCV-neo-replicon using as a facilitator $3 \mu \mathrm{l}$ of lipofectamine (Invitrogen) in 200 $\mu \mathrm{l}$ of Optimem (Gibco). At 5 hours post transfection, medium was replaced with DMEM containing $10 \%$ fetal calf serum and antibiotics. Replicon harboring cells were selected with G-418 (Roche) at a concentration of 250 $\mu \mathrm{g} / \mathrm{ml}$ for 20 days. Single cell clones obtained by endpoint dilution were grown and tested by PCR and Southern blot for the (lack of) incorporation of the Neomycin resistance gene into the genome and by Northern blot for the presence of the RNA transcript corresponding to the replicon (not shown). Expression of $\mathrm{HCV}$ protein was assessed by immunfluorscence using an anti-NS5a antibody (not shown). Clone D, which had high levels of HCV protein, harbored the HCV replicon, and did not have the Neo gene integrated into the cellular genome, was selected for further analysis. Clone D was "cured" from the replicon using a strategy similar as the one previously described [6]. Briefly, Clone D cells were passed four times at 7 or 8 day interval in absence of G-418 and treated with human Interferon (Sigma I2396) at a concentration of $100 \mathrm{IU} / \mathrm{ml}$. After two weeks, cells were tested for the absence of the $\mathrm{HCV}$ replicon by RT-PCR and their susceptibility to G-418. The expected PCR band was not detected, and cells regained susceptibility to G-418 at a concentration of $250 \mu \mathrm{g} / \mathrm{ml}$, which indicated that the Clone D cells were cured from the $\mathrm{HCV}$ replicon, and were named Huh7D cells. 


\section{Transfection of Huh7 cells with HCV replicons}

Huh7, Huh7D, and Huh7.5 cells grown in 12 well plates were transfected with different amounts of HCV-neo- or HCV-hyg replicons using as a facilitator $3 \mu \mathrm{l}$ of lipofectamine (Invitrogen) in $200 \mu \mathrm{l}$ of Optimem (Gibco). At 5 hours post transfection, medium was replaced with DMEM containing $10 \%$ fetal calf serum and antibiotics. At 24 hours post transfection, medium was replaced with same medium containing G-418 (Roche) at a concentration of $250 \mu \mathrm{g} / \mathrm{ml}$ (for HCV-neo-replicon transfected cells) or hygromycin B (Roche) at a concentration of $65 \mu \mathrm{g} / \mathrm{ml}$ (for HCV-hyg-replicon transfected cells). To measure susceptibility to HCV replication, the HCVneo-replicon transfected cells were fixed 13 or 15 days post transfection and stained with a solution of $50 \%$ methanol and $10 \%$ acetic acid containing $0.6 \mathrm{~g} / \mathrm{L}$ of Comassie brilliant blue. The HCV-hyg-replicon transfected cells were split in medium containing $65 \mu \mathrm{g} / \mathrm{ml}$ hygromycin $\mathrm{B}$, and colonies were stained with anti-HCV specific antibody as described below.

\section{Detection of HCV antigen by immunfluorscence (IF)}

Cells transfected with HCV-hyg-replicon or infected with HCV J6/JFH1 were fixed with methanol, blocked with a solution containing $1 \%$ BSA and $0.2 \%$ non-fat milk in $1 \times$ PBS, treated with a 1:200 dilution in $0.05 \%$ tween 20 in $1 \times$ PBS of a serum from a persistently infected chimpanzee that carried high levels of anti$\mathrm{HCV}$ antibodies [20] for 2 hours, washed with $1 \times \mathrm{PBS}$, stained with FITC-conjugated goat anti-human antibody (KPL), washed, and observed in the microscope.

\section{Infection of Huh7 cells with HCV J6/JFH1 and titration of progeny virus}

Huh7, Huh7D, and Huh7.5 cells grown in 6-well plates were mock infected or infected with $\mathrm{HCV}-\mathrm{J} 6 / \mathrm{JFH} 1$ at an moi of 0.01 . At 6 hours post infection, cells were washed three times with DMEM containing 10\% FCS and split into 12-well plates (for titration of total progeny virus) and 96-well plates (for IF analysis, described above). At $0,1,3,5,7$, and $10 \mathrm{dpi}$, the 12 -well plates were frozen at $-70^{\circ} \mathrm{C}$. For later time points, cells in one 12-well plate were split and treated as described above. Total virus from each time point was recovered by freezing and thawing the cells 3 times. Viral titers were obtained in Huh7.5 cells infected with 10-fold serial dilutions of the cell extracts followed by detection of viral antigens by IF analysis at three days post-infection as described above.

\section{Amplification and sequencing of RIG-I mRNA}

Total RNA was extracted from Huh7, Huh7D, and Huh7.5 cells grown in T25 flasks using Trizol reagent as recommended by the manufacturer (Invitrogen). cDNA was synthesized using $4 \mu \mathrm{g}$ of each RNA, SuperScript III reverse transcriptase (Invitrogen), and random primers. PCR amplification of RIG-I transcripts was performed using RIG-I specific primers RIG-I 91+ (5'CTACCCGGCTTTAAAGCTAG-3 and RIG-I 3020- (5'CGATCCATGATTATACCCAC-3'). Nested-PCR was performed using RIG-I-specific primers RIG-I 121+ (5'CCTGCGGGGAACGTAGCTAG-3') and RIG-I 530(5'-AATGATATCGGTTGGGATAA-3'), RIG-I 421+ (5'CCATTGAAAGTTGGGATTTC-3') and RIG-I 1410(5'-TGGCATCCCCAACACCAACC-3'), RIG-I 421+ and RIG-I 2990-(5'-TCTTCTCCACTCAAAGTTAC-3'). The Expand High Fidelity system (Roche) was used for PCR amplifications as described by the manufacturer. PCR products were run in agarose gels and purified using gene-elute agarose gel columns (Sigma) and sequenced (ABI-prism) using the above mentioned oligos and oligos RIG-I 474- (5'-GTAATCTATACTCCTCCAAC-3'), RIG-I 1331 - (5'-AGATCAGAAACTTGGAGGAT-3'), RIG-I 2281+ (5'-AGTGCAATCTTGTCATCCTT-3'), and RIG-I 2360- (5'-TCTTGCTCTTCCTCTGCCTC-3').

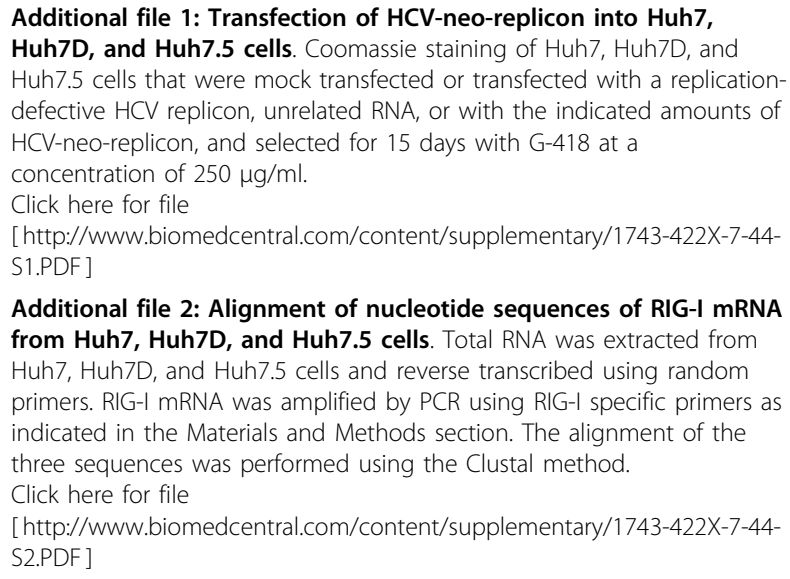

[http://www.biomedcentral.com/content/supplementary/1743-422X-7-44S1.PDF ]

Additional file 2: Alignment of nucleotide sequences of RIG-I mRNA from Huh7, Huh7D, and Huh7.5 cells. Total RNA was extracted from Huh7, Huh7D, and Huh7.5 cells and reverse transcribed using random primers. RIG-I mRNA was amplified by PCR using RIG-I specific primers as indicated in the Materials and Methods section. The alignment of the three sequences was performed using the Clustal method.

Click here for file

[ http://www.biomedcentral.com/content/supplementary/1743-422X-7-44S2.PDF ]

\section{Acknowledgements}

This work was supported by internal funding from the Food and Drug Administration. We thank Dr Charles Rice for providing the J6/JFH1 CDNA, Dr Ralf Bartenschlager for providing the pFK i389neoNS3-3'/NK5.1, pFK i389neoNS3-3'/delta5B, and pFK i389neoNS3-3'/wt plasmids, Dr Jake Liang for providing the Huh7 and Huh7.5 cells, and Dr Takaji Wakita for providing the JFH1 virus.

\section{Author details}

${ }^{1}$ Division of Viral Products, Center for Biologics Evaluation and Research, FDA, 29 Lincoln Drive, Bethesda, MD 20892, USA. Division of Emerging and Transfusion Transmitted Diseases, Center for Biologics Evaluation and Research, FDA, 29 Lincoln Drive, Bethesda, MD 20892, USA.

\section{Authors' contributions}

DF, GK, and SF conceived the study, its design, and coordination. DF isolated the Huh7D cells, characterized them, and generated the newly reported HCV-hyg replicons. DF performed the transfections and immunoassays. DF and KM performed the growth curves for the virus in the three cell lines. DF drafted the manuscript with the help of SF, GK, and KM. All authors approved the final version. 


\section{Competing interests}

The authors declare that they have no competing interests.

Received: 26 October 2009

Accepted: 19 February 2010 Published: 19 February 2010

\section{References}

1. Alter $H J$, Seeff $L B$ : Recovery, persistence, and sequelae in hepatitis $C$ virus infection: A perspective on long-term outcome. Seminars in Liver Disease 2000, 20:17-35.

2. Saito I, Miyamura T, Ohbayashi A, Harada H, Katayama T, Kikuchi S, et al: Hepatitis-C Virus-Infection Is Associated with the Development of Hepatocellular-Carcinoma. Proceedings of the National Academy of Sciences of the United States of America 1990, 87:6547-6549.

3. Alter H, Seeff LB: Viral Hepatitis Edinburgh: Churchill LivingstoneZuckermanm AZ, Thomas HC 1997, 467-498.

4. Wakita T, Pietschmann T, Kato T, Date T, Miyamoto M, Zhao ZJ, et al: Production of infectious hepatitis $\mathrm{C}$ virus in tissue culture from a cloned viral genome. Nature Medicine 2005, 11:791-796.

5. Sainz B Jr, Barretto N, Uprichard SL: Hepatitis C virus infection in phenotypically distinct Huh7 cell lines. PLoS One 2009, 4:e6561.

6. Blight $\mathrm{KJ}$, McKeating JA, Rice CM: Highly permissive cell lines for subgenomic and genomic hepatitis C virus RNA replication. Journal of Virology 2002, 76:13001-13014.

7. Zhong J, Gastaminza P, Cheng GF, Kapadia S, Kato T, Burton DR, et al: Robust hepatitis C virus infection in vitro. Proceedings of the National Academy of Sciences of the United States of America 2005, 102:9294-9299.

8. Yi M, Villanueva RA, Thomas DL, Wakita T, Lemon SM: Production of infectious genotype 1a hepatitis $C$ virus (Hutchinson strain) in cultured human hepatoma cells. Proceedings of the National Academy of Sciences of the United States of America 2006, 103:2310-2315.

9. Lindenbach BD, Evans MJ, Syder AJ, Wolk B, Tellinghuisen TL, Liu CC, et al: Complete replication of hepatitis C virus in cell culture. Science 2005, 309:623-626.

10. Pietschmann T, Zayas M, Meuleman P, Long G, Appel N, Koutsoudakis G, et al: Production of Infectious Genotype 1b Virus Particles in Cell Culture and Impairment by Replication Enhancing Mutations. Plos Pathogens 2009, 5.

11. Tanner NK, Linder P: DExD/H box RNA helicases: From generic motors to specific dissociation functions. Molecular Cell 2001, 8:251-262.

12. Sumpter R, Loo YM, Foy E, Li K, Yoneyama M, Fujita T, et al: Regulating intracellular antiviral defense and permissiveness to hepatitis $C$ virus RNA replication through a cellular RNA helicase, RIG-I. Journal of Virology 2005, 79:2689-2699.

13. Binder $M$, Kochs $G$, Bartenschlager $R$, Lohmann V: Hepatitis $C$ virus escape from the interferon regulatory factor 3 pathway by a passive and active evasion strategy. Hepatology 2007, 46:1365-1374.

14. Wang N, Liang Y, Devaraj S, Wang J, Lemon SM, Li K: Toll-like receptor 3 mediates establishment of an antiviral state against hepatitis $\mathrm{C}$ virus in hepatoma cells. J Virol 2009, 83:9824-9834.

15. Borawski J, Troke P, Puyang X, Gibaja V, Zhao S, Mickanin C, et al: Class III phosphatidylinositol 4-kinase alpha and beta are novel host factor regulators of hepatitis C virus replication. J Virol 2009, 83:10058-10074.

16. Randall G, Panis M, Cooper JD, Tellinghuisen TL, Sukhodolets KE, Pfeffer S, et al: Cellular cofactors affecting hepatitis $C$ virus infection and replication. Proceedings of the National Academy of Sciences of the United States of America 2007, 104:12884-12889.

17. Ng Tl, Mo H, Pilot-Matias T, He Y, Koev G, Krishnan P, et al: Identification of host genes involved in hepatitis $C$ virus replication by small interfering RNA technology. Hepatology 2007, 45:1413-1421.

18. Lohmann V, Korner F, Koch JO, Herian U, Theilmann L, Bartenschlager R: Replication of subgenomic hepatitis $C$ virus RNAs in a hepatoma cell line. Science 1999, 285:110-113.

19. Krieger N, Lohmann V, Bartenschlager R: Enhancement of hepatitis $C$ virus RNA replication by cell culture-adaptive mutations. Journal of Virology 2001, 75:4614-4624.

20. Major ME, Mihalik K, Fernandez J, Seidman J, Kleiner D, Kolykhalov AA, et al: Long-term follow-up of chimpanzees inoculated with the first infectious clone for hepatitis C virus. Journal of Virology 1999, 73:3317-3325.
doi:10.1186/1743-422X-7-44

Cite this article as: Feigelstock et al:: Increased susceptibility of Huh7 cells to HCV replication does not require mutations in RIG-I. Virology Journal 2010 7:44.

\section{Submit your next manuscript to BioMed Central and take full advantage of:}

- Convenient online submission

- Thorough peer review

- No space constraints or color figure charges

- Immediate publication on acceptance

- Inclusion in PubMed, CAS, Scopus and Google Scholar

- Research which is freely available for redistribution

Submit your manuscript at www.biomedcentral.com/submit
Ciomed Central 